\title{
Antagonism of miR-33 in mice promotes reverse cholesterol transport and regression of atherosclerosis
}

\author{
Katey J. Rayner, ${ }^{1}$ Frederick J. Sheedy, ${ }^{1}$ Christine C. Esau, ${ }^{2}$ Farah N. Hussain, ${ }^{1}$ Ryan E. Temel, ${ }^{3}$ \\ Saj Parathath, ${ }^{1}$ Janine M. van Gils, ${ }^{1}$ Alistair J. Rayner, ${ }^{1}$ Aaron N. Chang, ${ }^{2}$ Yajaira Suarez, ${ }^{1}$ \\ Carlos Fernandez-Hernando,, ${ }^{1,4}$ Edward A. Fisher, ${ }^{1}$ and Kathryn J. Moore ${ }^{1,4}$ \\ ${ }^{1}$ Marc and Ruti Bell Vascular Biology and Disease Program, Leon H. Charney Division of Cardiology, Department of Medicine, \\ New York University School of Medicine, New York, New York, USA. ${ }^{2}$ Regulus Therapeutics, San Diego, California, USA. \\ 3Department of Pathology, Section on Lipids Sciences, Wake Forest University School of Medicine, Winston-Salem, North Carolina, USA. \\ ${ }^{4}$ Department of Cell Biology, New York University School of Medicine, New York, New York, USA.
}

\begin{abstract}
Plasma HDL levels have a protective role in atherosclerosis, yet clinical therapies to raise HDL levels have remained elusive. Recent advances in the understanding of lipid metabolism have revealed that miR-33, an intronic microRNA located within the SREBF2 gene, suppresses expression of the cholesterol transporter ABC transporter A1 (ABCA1) and lowers HDL levels. Conversely, mechanisms that inhibit miR-33 increase ABCA1 and circulating HDL levels, suggesting that antagonism of miR-33 may be atheroprotective. As the regression of atherosclerosis is clinically desirable, we assessed the impact of miR-33 inhibition in mice deficient for the LDL receptor $\left(\mathrm{Ldll}^{-/-}\right.$mice), with established atherosclerotic plaques. Mice treated with anti-miR33 for 4 weeks showed an increase in circulating HDL levels and enhanced reverse cholesterol transport to the plasma, liver, and feces. Consistent with this, anti-miR33-treated mice showed reductions in plaque size and lipid content, increased markers of plaque stability, and decreased inflammatory gene expression. Notably, in addition to raising ABCA1 levels in the liver, anti-miR33 oligonucleotides directly targeted the plaque macrophages, in which they enhanced ABCA1 expression and cholesterol removal. These studies establish that raising HDL levels by anti-miR33 oligonucleotide treatment promotes reverse cholesterol transport and atherosclerosis regression and suggest that it may be a promising strategy to treat atherosclerotic vascular disease.
\end{abstract}

\section{Introduction}

Plasma HDL cholesterol (HDL-C) levels bear a strong inverse relationship with cardiovascular disease risk, and, as such, therapies to raise HDL are being actively pursued (1). There is strong evidence that high levels of circulating HDL are associated with positive cardiovascular outcomes, independent of levels of LDL cholesterol (LDL-C). According to the Framingham Heart Study, for every $1 \%$ increase in circulating HDL-C, there is a $2 \%$ decrease in overall risk of developing coronary heart disease (2). In mouse models of atherosclerosis, overexpression of apoA1 to increase HDL has been shown to hinder plaque progression (3-6) and to promote regression $(7,8)$. Furthermore, direct infusion of HDL in apoE-deficient mice (9), cholesterol-fed rabbits (10), or human subjects (11) with established atherosclerosis, reduces plaque size. Such studies strongly suggest that HDL-raising strategies may be an effective therapy for the treatment of atherosclerosis. Despite this, our understanding of the mechanisms that contribute to HDL biogenesis and regulation, and how to effectively manipulate these for therapeutic potential, remain largely incomplete.

The identification of $\mathrm{ABC}$ transporter $\mathrm{A} 1$ ( $A B C A 1)$ as the gene mutated in Tangier disease, a disease characterized by low levels of circulating HDL, revealed the critical role of ABCA1 in the gen-

Conflict of interest: Edward A. Fisher is a member of a Merck advisory board and has received honoraria from Merck for speaking engagements. Christine C. Esau and Aaron N. Chang are employees of Regulus Therapeutics, which develops microRNAtargeted therapies.

Citation for this article: J Clin Invest. 2011;121(7):2921-2931. doi:10.1172/JCI57275 eration of HDL particles $(12,13)$. ABCA1 transports free cholesterol from within the cell to lipid-poor apoA1 particles to generate nascent HDL, a function critical to the biogenesis of HDL in the liver and for the efflux of excess cholesterol from cells in peripheral tissues (14). Acting in concert with ABCA1, another member of the $\mathrm{ABC}$ family of transporters, ABCG1, is responsible for cellular cholesterol efflux to further lipidate HDL, generating larger $\alpha$-HDL particles that are destined for clearance by the liver (15-17). In vivo molecular-genetic causation studies indicate that together ABCA1 and ABCG1 have the major role in mediating net cholesterol efflux from macrophages to HDL (18-21). This combined process, termed reverse cholesterol transport (RCT), mediates clearance of excess cholesterol from cells to the liver for excretion to the bile and feces. This process is particularly relevant to the removal of cholesterol from lipid-engorged foam cells that accumulate in atherosclerotic lesions and is thought to contribute prominently to the atheroprotective effects of HDL.

The expressions of both ABCA1 and ABCG1 are upregulated in states of cholesterol excess by the liver $\mathrm{X}$ receptor (LXR) nuclear hormone transcription factors. LXRs are activated by oxysterol metabolites of cholesterol and play key roles in regulating multiple components of the RCT pathway (22), cholesterol conversion to bile acid, and intestinal cholesterol absorption. Studies in mouse models of both atherosclerosis progression and regression have shown that synthetic LXR agonists have potent antiatherosclerotic effects (23-27). However, while very effective in preclinical studies, all LXR agonists developed to date have a substantial caveat that currently precludes their translation into human clinical trials. Specifically, LXR activa- 

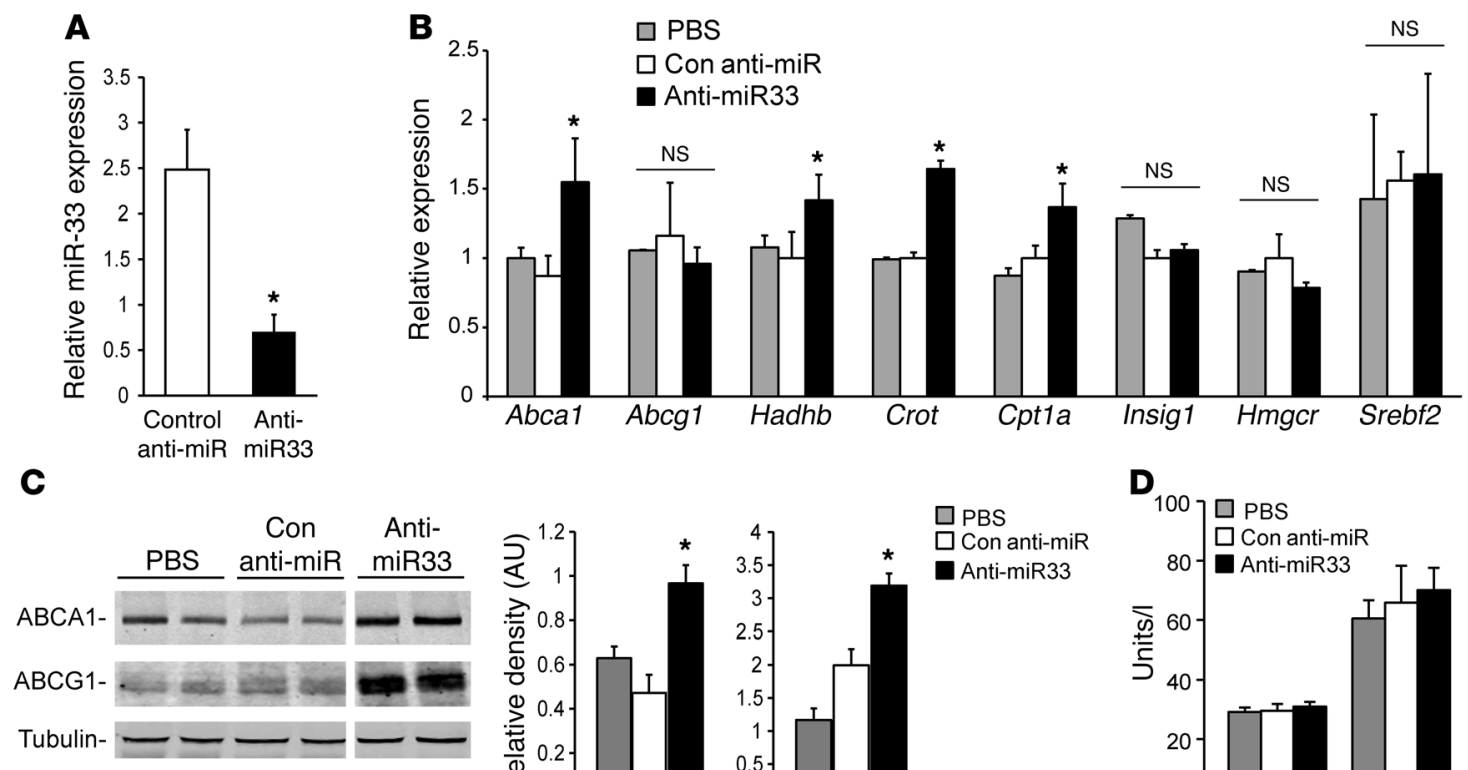

Figure 1
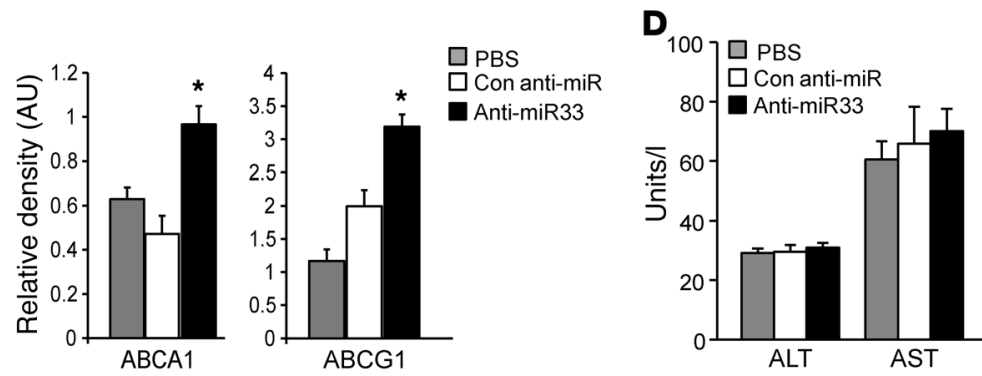

Anti-miR33 treatment reduces miR-33 expression in the liver and causes derepression of its target genes. Ldlr $r^{-1-}$ mice were fed a WD for 14 weeks and then subsequently treated for 4 weeks with PBS (no treatment) or control anti-miR or anti-miR33 2'F/MOE oligonucleotides. (A and B) Expression of (A) miR-33, (B) miR-33 target genes (Abca1, Abcg1, Hadhb, Crot, Cpt1a), and nontarget lipid metabolism genes (Insig1, Hmgcr, Srebf2) in the liver was quantified by RT-PCR. Con, control. (C) Western blot of hepatic ABCA1 and ABCG1 protein. The white line denotes lanes run noncontiguously. ${ }^{*} P \leq 0.05$, compared with controls. (D) Plasma levels of ALT and AST.

tion of fatty acid synthase and SREBP-1c transcription in the liver leads to hypertriglyceridemia and hepatic steatosis. As a result, the therapeutic potential of the ABCA1 pathway remains untapped.

Recently, our group and others reported that the SREBP-2 locus transcribes not only the mRNA for this transcriptional regulator of cholesterol uptake and synthesis but also a highly conserved microRNA, miR-33a, that represses multiple genes involved in cellular cholesterol trafficking (28-30). In humans and nonhuman primates, a related microRNA, miR-33b, is encoded in the SREBP-1 locus. The 3 ' untranslated region (UTR) of $A b c a 1$ contains 3 highly conserved binding sites for miR-33, and the expression of $A B C A 1$ mRNA and ABCA1 protein is strongly repressed by miR-33 overexpression in a variety of cell types (28-30). Functionally, overexpression of miR-33 in hepatocytes and macrophages decreases cholesterol efflux to apoA1 - a key step in the generation of HDL and RCT. Moreover, the inhibition of endogenous miR-33 results in an increase in expression of $\mathrm{ABCA} 1$ protein and cholesterol efflux to apoA1, indicating a physiologically relevant role for this miRNA in regulating ABCA1 (28-30). In mice, but not humans, miR-33 also targets ABCG1 and reduces efflux of cholesterol to HDL (28). Notably, in vivo antagonism of miR-33 in mice, using a variety of methods ranging from lentiviral-mediated antisense inhibition to complete genetic deletion, results in a significant increase in circulating HDL-C (28-30). These data suggest that miR-33 may be an attractive therapeutic target for the treatment of cardiovascular disease; however, several questions remain to be addressed, particularly regarding the functionality of the HDL produced and its ability to promote RCT.

In the current study, we tested the impact of miR-33 inhibition on $\mathrm{RCT}$ and atherosclerosis in the hypercholesterolemic mouse model deficient for the LDL receptor ( $\mathrm{Ldlr}^{-/}$mice). As patients in a clini- cal setting typically present with already established atherosclerosis, therapies that can reverse disease will likely be most attractive. Thus, to test the efficacy of anti-miR33 in a model setting that most closely approximates this, we first established atherosclerosis in $\mathrm{Ldll}^{-/}$- mice by feeding them a Western diet (WD), after which they were treated weekly for 4 weeks with anti-miR33 or control anti-miR oligonucleotides. We demonstrated herein that inhibition of miR-33 expression effectively raises circulating HDL, increases RCT, and decreases atherosclerotic plaque size. Furthermore, characterization of atherosclerotic lesions in anti-miR33-treated mice showed increased markers of plaque stability, including reduced macrophage and lipid accumulation, decreased expression of inflammatory genes, and increased collagen content. These data establish that inhibition of endogenous miR-33 to therapeutically raise HDL enhances RCT and induces regression of atherosclerosis.

\section{Results}

Anti-miR33 increases expression of miR-33 target genes in the liver, including ABCA1. The subcutaneous or intraperitoneal delivery of $2^{\prime}$ fluoro/methoxyethyl-modified ( $2^{\prime} \mathrm{F} / \mathrm{MOE}$-modified) phosphorothioate backbone antisense oligonucleotides has been used successfully in mice to inhibit the function of various miRNAs and to increase expression of their target genes, with no apparent toxicity (31). Similar anti-miRNA strategies have been shown to be well tolerated in nonhuman primates $(32,33)$, indicating that these antisense oligonucleotides may be promising therapeutic agents. To assess the effects of inhibiting miR-33 in a model of established atherosclerosis, $\mathrm{Ldlr}^{-/}$- mice were first fed a WD for 14 weeks (baseline), after which they were switched to a chow diet to block atherosclerosis progression and injected subcutaneously with $10 \mathrm{mg} / \mathrm{kg}$ of $2^{\prime} \mathrm{F} / \mathrm{MOE}$ anti-miR33 or control anti-miR oligonucleotides or 
A

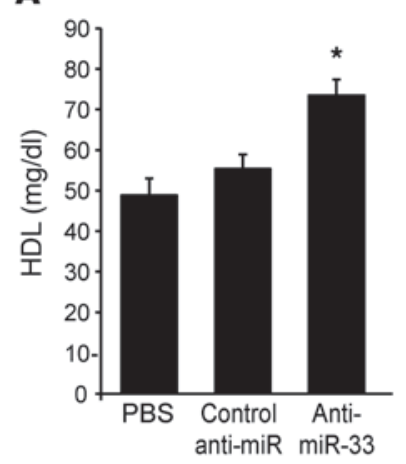

B

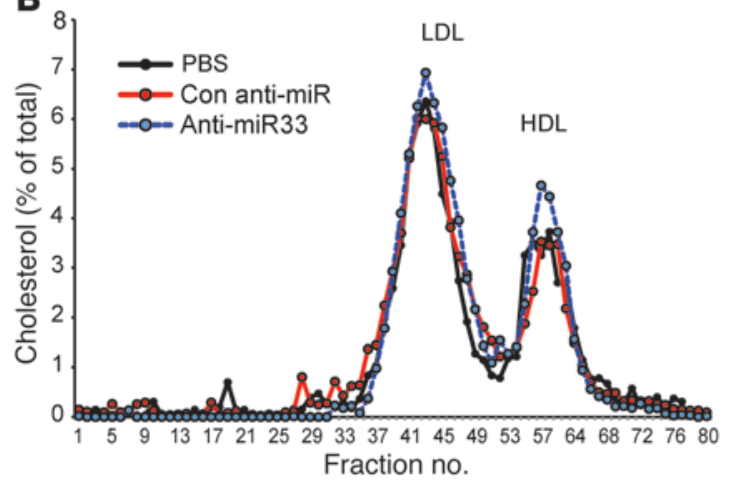

C

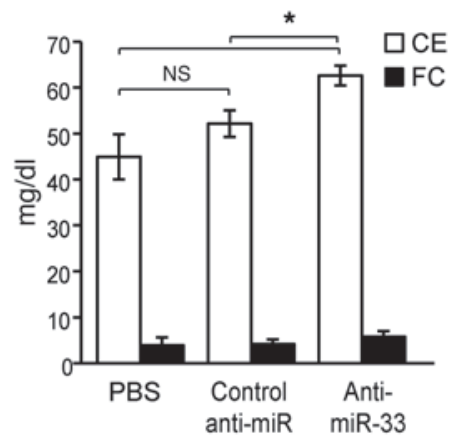

D

Fraction: $53-55 \quad 56-58 \quad 59-61 \quad 62-64 \quad 65-67 \quad 68-70$
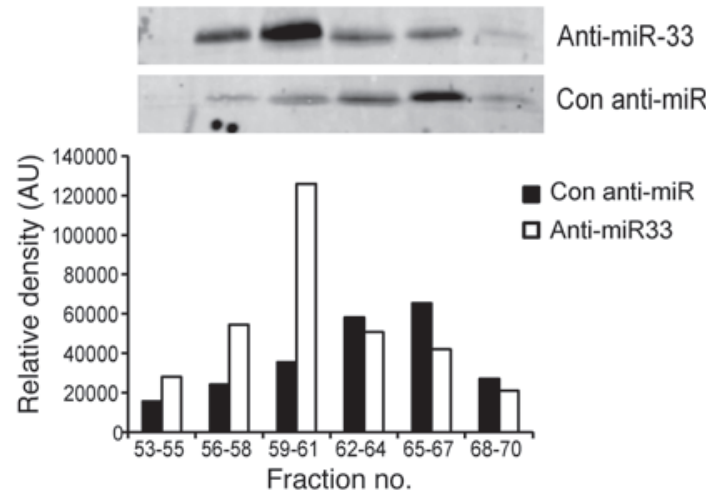

Con anti-miR

anti-miR33

apoE

Fraction: $53-55 \quad 56-58 \quad 59-61 \quad 62-64 \quad 65-67 \quad 68-70$
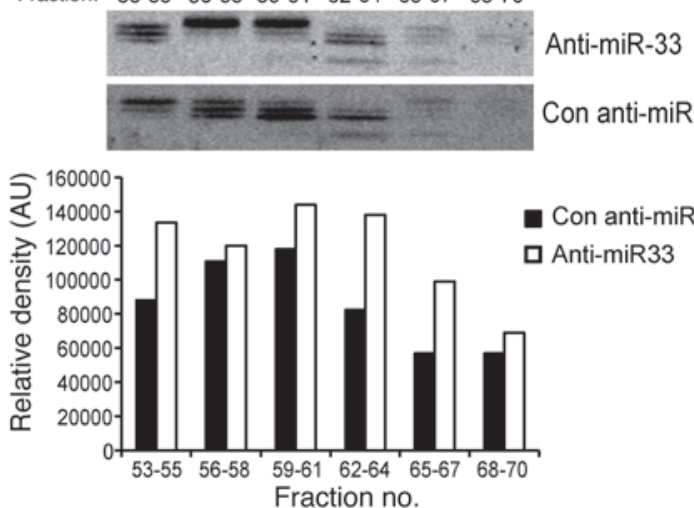

Figure 2

Anti-miR33 increases circulating HDL in $L d l r^{-1}$ mice. (A) Plasma HDL levels after 4 weeks of the indicated treatment $\left(n=15\right.$ mice/group). ${ }^{*} P \leq 0.05$, compared with controls. (B) FPLC lipoprotein profiles from pooled plasma $(n=4)$ of PBS-treated and control anti-miR and anti-miR33-treated mice. (C) Cholesterol ester (CE) and free cholesterol (FC) content of the HDL. ${ }^{*} P \leq 0.05$, compared with controls. (D) Western blots of apoA1 and apoE in HDL lipoprotein fractions (fraction number 53-70 of FPLC profile).

PBS (untreated). To maximize anti-miR delivery, mice were injected twice during the first week, then once weekly thereafter, for a total of 4 weeks. Consistent with previous studies (34), this regimen did not induce a detectable immune response, as serum levels of IL- 6 and monocyte chemoattractant protein 1 (MCP-1) in mice treated with either control anti-miR or anti-miR33 did not differ from those of mice injected with PBS alone (data not shown).

To determine the efficacy of anti-miR33 treatment, we measured the expression of miR-33 and its target genes in the livers of mice after 4 weeks of treatment. Levels of miR-33, detected by quantitative RT-PCR, were decreased by more than $60 \%$ in anti-miR33treated mice compared with those of mice receiving control antimiR (Figure 1A). Consistent with this, the expression of ABCA1 in the liver was increased or "derepressed" in the anti-miR33-treated group compared with that in untreated or control anti-miRtreated mice; however, no change in $A b c g 1$ mRNA was observed (Figure 1B). Furthermore, both ABCA1 and ABCG1 protein were increased in the livers of anti-miR33-treated mice, compared with those of control groups (Figure 1C). To confirm the specificity of anti-miR33 action, we examined the expression of other hepatic lipid metabolism genes. While there were no significant changes in genes lacking functional miR-33 target sites (i.e., Insig1, Hmgcr, and Srebf2), the expression of other known miR-33 target genes involved in fatty acid metabolism was significantly increased with anti-miR33 treatment (Crot, Hadbb, Cpt1a) (Figure 1B). Importantly, no elevation in serum hepatotoxicity markers, aspartate aminotransferase and alanine aminotransferase (AST and ALT), was noted in control anti-miR- or anti-miR-33-treated mice as compared with that in mice treated with PBS (Figure 1D). Together these data demonstrate that $2^{\prime} \mathrm{F} / \mathrm{MOE}$ anti-miR33 effectively inhibits miR-33 activity and selectively increases expression of genes repressed by miR-33.

Anti-miR33 treatment increases HDL and enhances RCT in vivo. As increased ABCA1 expression in the liver would be predicted to augment HDL biogenesis, we measured circulating total and HDL-C levels in the anti-miR33- and control anti-miR-treated mice. Consistent with previous work by our laboratory and others showing that short-term (5-12 days) inhibition of miR-33 in C57BL/6 mice results in $25 \%-30 \%$ increases in circulating HDL (28-30), treatment of $\mathrm{Ldlr}^{-/}$mice with anti-miR33 for 4 weeks raised HDL-C by $35 \%$ compared with that of control mice (Figure 2A). By contrast, there was no difference in total circulating cholesterol among mice that were treated with control anti-miR, anti-miR33, or PBS (Table 1). Analysis of lipoproteins by fast protein liquid chromatography (FPLC) showed an increase in cholesterol content of the HDL fractions (fractions 54-67) of the anti-miR33-treated mice compared with that of control anti-miR- or PBS-treated mice (Figure 2B). Further analysis of the free cholesterol and cholesterol ester con- 
Table 1

Lipid and body weight parameters

$\begin{array}{lcccc} & \text { Baseline } & \text { PBS } & \text { Control anti-miR } & \text { Anti-miR33 } \\ \text { Starting body weight }(\mathrm{g}) & - & 27.4 \pm 2.1 & 30.4 \pm 2.2 & 31.5 \pm 2.5 \\ \text { Body weight at sacrifice }(\mathrm{g}) & 27.2 \pm 2.0 & 26.6 \pm 0.65 & 24.5 \pm 1.4 & 24.9 \pm 1.6 \\ \text { Total cholesterol }(\mathrm{mg} / \mathrm{dl}) & 1,089 \pm 162 & 264 \pm 40 & 250 \pm 46 & 272 \pm 45 \\ \text { Plasma triglyceride }(\mathrm{mg} / \mathrm{dl}) & 263.8 \pm 34 & 56.5 \pm 15 & 64.9 \pm 19 & 71.2 \pm 18\end{array}$

Body weight was obtained at baseline (after 14w WD) and at sacrifice (after 4 weeks treatment with PBS, control anti-miR, or anti-miR33). Total plasma cholesterol and triglyceride levels were obtained at sacrifice. All data are expressed as mean \pm SD ( $n=15$ mice/group).

pathway by which excess cholesterol is effluxed from peripheral tissues, a process that is particularly important in the removal of cholesterol from atherosclerotic lesions.

Anti-miR33 treatment induces atherosclerosis regression and lesion remodeling. Data from mouse models of apoA1 overexpression or HDL infusion suggest that raising HDL-C favorably impacts atherosclerosis $(3-5,8)$. We thus hypothesized that the enhanced RCT in antimiR33-treated mice would promote

tent of the HDL revealed an increase in HDL-associated cholesterol ester in anti-miR-33-treated mice (Figure 2C). To test whether anti-miR33 treatment altered the apolipoprotein content on HDL particles, we measured apoA 1 and apoE in the HDL fractions by Western blotting. In anti-miR33-treated mice, there was an overall increase in apoA1 recovered in the HDL fractions compared with that in control anti-miR-treated mice (Figure 2D). Moreover, a higher proportion of the apoA1 was associated with larger HDL particles in anti-miR-33-treated mice. In addition, there appeared to be an increase in glycosylation of the apoE in the anti-miR-33treated mice. Together, these data are consistent with increased efflux of cholesterol from cells to HDL from peripheral tissues and maturation of the HDL particle.

To determine whether higher HDL levels in response to anti-miR33 treatment upregulates cholesterol transport from peripheral cells to the liver for further excretion into bile and feces, we performed an in vivo RCT assay that traces ${ }^{3} \mathrm{H}$-cholesterol from macrophages loaded with cholesterol ex vivo $(35,36)$. Anti-miR33-treated mice injected subcutaneously with cholesterol-loaded $/{ }^{3} \mathrm{H}$-cholesterol-labeled bone marrow-derived macrophages showed a $35 \%-40 \%$ increase in the appearance of ${ }^{3} \mathrm{H}$-cholesterol in plasma over 48 hours, compared with that of control mice (Figure 3A). Furthermore, anti-miR33-treated mice showed a $42 \%$ increase in the delivery of ${ }^{3} \mathrm{H}$-tracer to the liver (Figure 3B) and an $82 \%$ increase in ${ }^{3} \mathrm{H}$-sterols excreted into feces (Figure 3C). Together, these results establish that miR-33 inhibition not only increases circulating HDL, but enhances the RCT removal of cholesterol from vessel wall foam cells, leading to plaque regression. The mean aortic sinus lesion area of $\mathrm{Ldlr}^{-/-}$ mice injected for 4 weeks with either PBS (untreated) or control anti-miR did not differ from that of mice harvested after 14 weeks of WD feeding (baseline) (Figure 4A). By contrast, antimiR33-treated mice had a $35 \%$ reduction in lesion area in the aortic sinus compared with that of baseline and control groups (Figure 4A). Examination of hematoxylin- and eosin-stained cross sections of the aortic root showed that in addition to a reduction in size, plaques in anti-miR-33-treated mice appeared to have been substantially remodeled compared with plaques in control anti-miR-treated mice (Figure 4B). To further investigate this, we characterized markers of lesion composition and stability. Quantification of lipid accumulation in aortic sinus lesions by oil red $\mathrm{O}$ staining demonstrated a corresponding $28 \%$ decrease in lipid accumulation in anti-miR33-treated mice compared with that of controls (Figure 5A). Moreover, there was a $35 \%$ reduction in $\mathrm{CD} 68^{+}$macrophage content (Figure $5 \mathrm{~B}$ ) and a 2 -fold increase in total lesional collagen content in antimiR33-treated mice compared with that of controls (Figure 5C), indicating remodeling of plaques toward a more stable phenotype. No difference in lesional apoptosis, as measured by TUNEL-positive cells in plaques, was noted among the various treatment groups (data not shown). Together, these results indicate that anti-miR33 treatment supports the efflux of cholesterol from the lesional macrophages and promotes regression of established atherosclerosis.
A

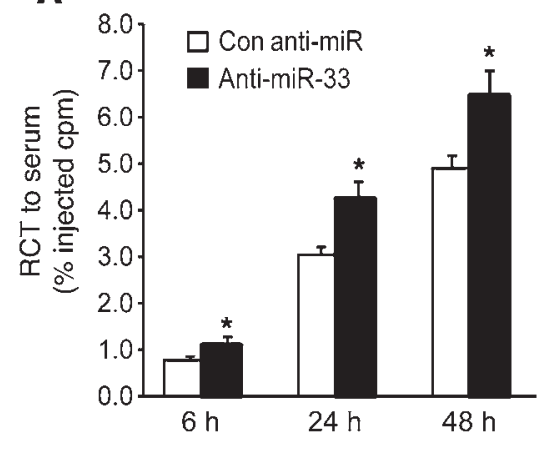

B

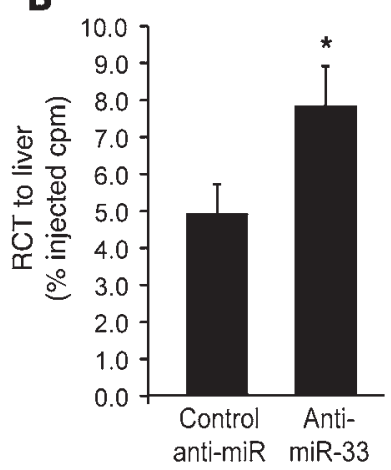

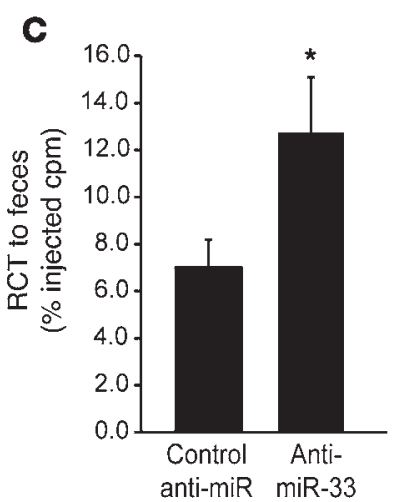

Figure 3

RCT is increased in anti-miR33-treated mice. After 4 weeks of the indicated, treatment $L d r^{-1}$ mice $(n=8 /$ group) were injected subcutaneously with ${ }^{3} \mathrm{H}$-cholesterol-labeled, acLDL-loaded bone marrow-derived macrophages. Data are expressed as the percentage of the ${ }^{3} \mathrm{H}$-cholesterol tracer relative to that of total cpm tracer injected \pm SD. (A) Time course of ${ }^{3} \mathrm{H}$-cholesterol distribution in plasma. (B) $\mathrm{Hepatic}{ }^{3} \mathrm{H}$-cholesterol tracer levels after 48 hours. (C) Fecal ${ }^{3} \mathrm{H}$-cholesterol tracer levels. Feces were collected continuously from 0 to 48 hours after injection. ${ }^{*} P \leq 0.05$, compared with controls. 
A

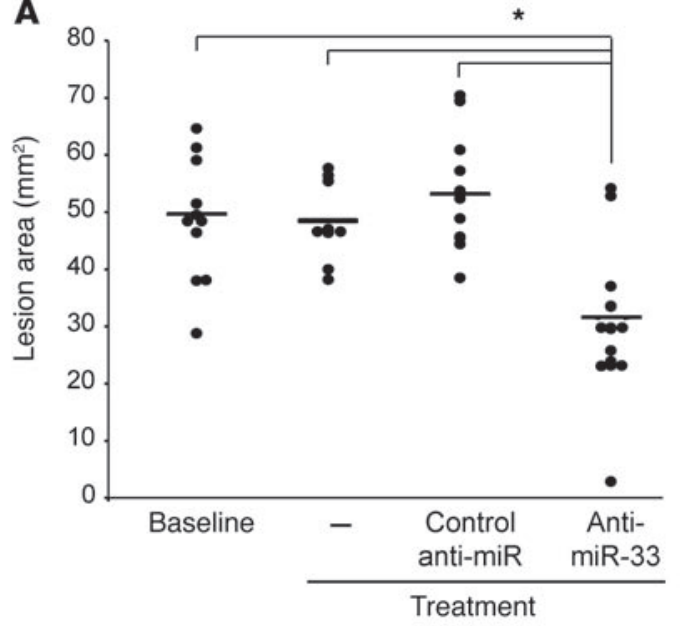

B

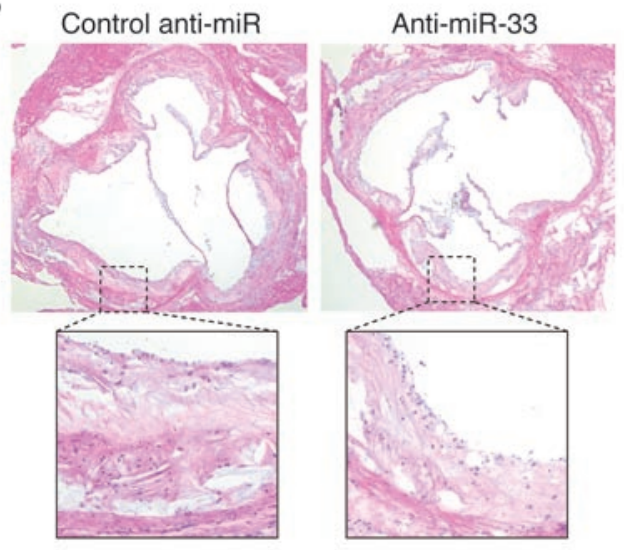

\section{Figure 4}

Anti-miR33 treatment regresses atherosclerosis. (A) Quantification of the lesion area of mice ( $n=15 / g r o u p)$ at baseline (14 weeks of WD) and after 4 weeks of the indicated treatment. Horizontal bars indicate the mean, and individual symbols indicate individual mice. PBS treatment is indicated by the dash. Data are the mean \pm SEM. ${ }^{*} \leq 0.05$. (B) Representative hematoxylin- and eosin-stained aortic sinus sections from control anti-miR- and anti-miR33-treated mice. Original magnification: $\times 50$ (top images); ×200 (bottom images).

Anti-miRNA oligonucleotides target lesional macrophages. Subcutaneous delivery of $2^{\prime} \mathrm{F} / \mathrm{MOE}$ oligonucleotides has been shown to reach a variety of tissues, including liver and spleen (37); however, it is not known whether these anti-miRNA molecules can reach the macrophages within the plaque to directly alter target gene expression in these cells. To test this, we performed immunohistochemical staining of aortic sinus lesions using an antibody directed against the phosphorothioate backbone of the 2'F/MOE oligonucleotides. We show that the anti-miRNA oligonucleotides were found within the plaque where they colocalize with $\mathrm{CD}^{+} 8^{+}$macrophages (Figure 6A). This technique detected both the control anti-miR and anti-miR33 oligonucleotides in lesional macrophages. To determine whether anti-miR33 affects target gene expression within the plaque, we isolated lesional $\mathrm{CD} 8^{+}$macrophages using laser-capture microdissection and extracted RNA for gene expression analysis. Examination of ABCA1 expression levels in lesional macrophages demonstrated similar levels of $A b c a 1$ mRNA in baseline, PBS-treated, or control anti-miR-treated mice (Figure 6B). Notably, anti-miR33treated mice had a $66 \%$ increase in lesional macrophage Abca1 expression compared with these control groups (Figure 6B). This is the first evidence to our knowledge that $2^{\prime} \mathrm{F} / \mathrm{MOE}$ anti-miRNA oligonucleotides are capable of penetrating the atherosclerotic lesion to reach plaque macrophages, in which they can directly alter target gene expression.

To further understand the impact of anti-miR33 on the plaque macrophage phenotype, we performed gene expression profiling of RNA isolated from lesional macrophages using Affymetrix gene arrays. Cumulative distribution function (CDF) analysis revealed that treatment with anti-miR33 resulted in a statistically significant enrichment in the expression of genes containing miR-33 binding sites in their $3^{\prime}$ UTR compared with that in non-miR33 target genes (Figure 6C; $P=2.02 \times 10^{-4}$ ), consistent with specific derepression of miR-33 targets in lesional macrophages. In addition, gene ontology analysis showed a significant downregulation in genes involved in the immune response in plaque macrophages from anti-miR33-treated mice compared with control anti-miR- treated mice $\left(\right.$ Benjamini-corrected $P$ value $=8.1 \times 10^{-3}$; Supplemental Table 1; supplemental material available online with this article; doi:10.1172/JCI57275DS1). Quantitative PCR confirmed downregulation of several of these inflammatory genes in lesional macrophage of anti-miR33-treated mice, including Tnfa, Tlr6, and Tlr13 (Figure 7A). Recent evidence suggests that regressing atherosclerotic lesions contain a greater proportion of macrophages characterized by the reparative M2 phenotype compared with that of the proinflammatory M1 macrophages and that HDL can promote regression in part by enhancing this pathway (38). In accordance with this, we found that lesional macrophages from anti-miR33-treated mice show increased mRNA levels of antiinflammatory M2 markers (Arg1, Il10) and reduced expression of proinflammatory M1 markers (iNos and Tnfa). Overall, these gene expression analyses suggest that in addition to containing less lipid and increased markers of plaque stability, macrophages from within the lesions of anti-miR33-treated mice have a less inflammatory and more reparative phenotype.

\section{Discussion}

The current study demonstrates that miR-33 inhibition in $\mathrm{Ldlr}^{-/}$ mice raises circulating HDL, enhances RCT, and promotes the regression of established atherosclerosis. Although previous reports, including one from our own group, showed that miR-33 antagonism increases hepatic ABCA1 and circulating HDL-C levels in C57BL/6 mice (28-30), this study demonstrates that the HDL generated by this method is functional and can promote removal of excess cholesterol from the periphery into the RCT pathway for excretion. This increase in RCT is very likely responsible for the favorable effects of anti-miR33 on atherosclerosis that we observe, including reductions in lesion area, macrophage number, lipid content, and inflammatory gene expression. Furthermore, as well as targeting the liver, our study shows for the first time to our knowledge that 2'F/MOE oligonucleotides penetrate the atherosclerotic plaque to reach lesional macrophages, in which they can upregulate ABCA1 expression and enhance cholesterol removal 
A

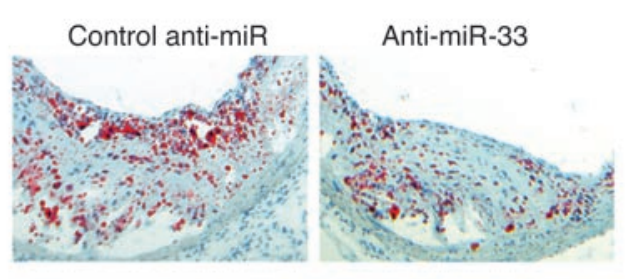

B

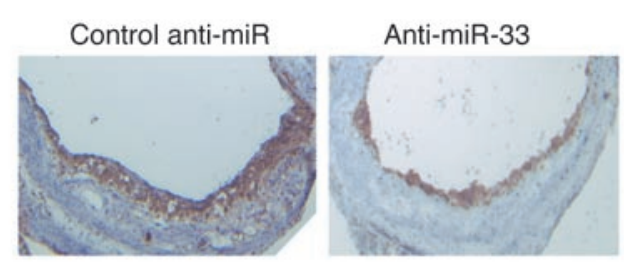

C

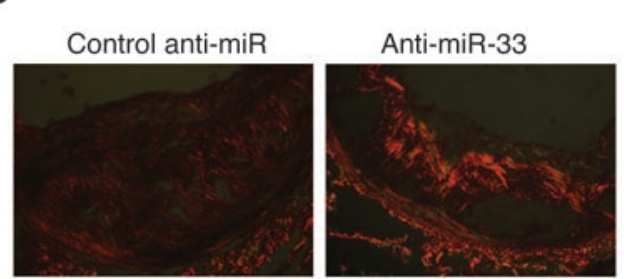

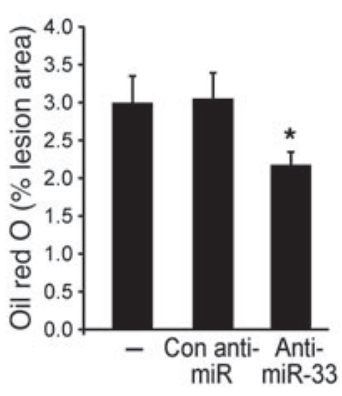
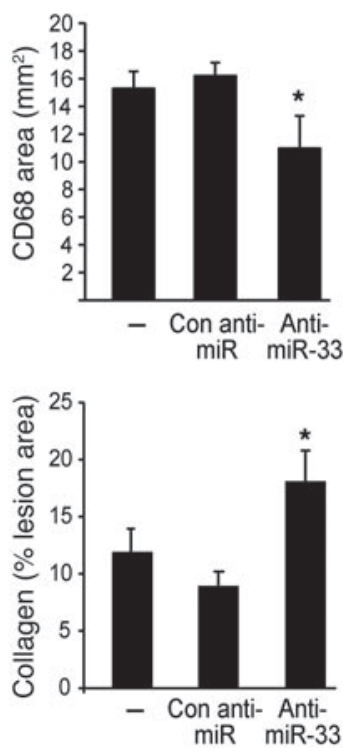

Figure 5

Inhibition of miR-33 improves markers of atherosclerotic plaque stability. Characterization of atherosclerotic lesion composition by $(\mathbf{A})$ oil red $\mathrm{O}$ staining for neutral lipids, (B) immunohistochemical staining for the macrophage marker CD68, and (C) picrosirius red staining for collagen. Images show representative sections from control and anti-miR33-treated mice, and quantification ( $n=10$ mice/group) is shown in the accompanying bar graph. ${ }^{*} P \leq 0.05$, compared with no treatment and control anti-miR. Original magnification: $\times 200(\mathbf{A}$ and C); $\times 100(B)$.

from foam cells. Therefore, not only does anti-miR33 function to raise HDL and promote RCT, but the direct targeting of the macrophages in the plaque upregulates cellular cholesterol efflux pathways to further enhance RCT and favorably affect lesion pathology. Together, these data establish miR-33 as an attractive therapeutic target for raising HDL and regressing atherosclerosis.

The recent generation of a miR-33 knockout mouse showed that loss of miR-33 in C57BL/ 6 mice increases HDL by up to $40 \%$, with a specific increase in larger HDL particles (39). Notably, subcutaneous delivery of $2^{\prime} \mathrm{F} / \mathrm{MOE}$ anti-miR33 oligonucleotides in the atherosclerotic $\mathrm{Ldlr}^{-/}$mouse model achieved an increase in HDL of a similar magnitude, indicating robust antagonism of endogenous miR-33 and target gene derepression. The levels of hepatic miR-33 detectable by quantitative RT-PCR (qRT-PCR) were greatly reduced in anti-miR33-treated mice compared with mice receiving control treatments, which is consistent with efficient delivery of anti-miR-33 to this tissue upon parenteral administration, as has previously been described for such modified oligonucleotides (37). However, as the accumulation of anti-miR-33 may overestimate the degree of inhibition of miR-33 expression by interfering with in vitro PCR amplification, a more relevant measure of efficacy is the expression of miR-33 target genes in this tissue. Indeed, in the livers of anti-miR33-treated mice, we saw increased expression of a number of validated miR-33 target genes, including the cholesterol transporters, $A b c a 1$ and $A b c g 1$ (28-30), and the fatty acid metabolism genes, Crot, Hadhb, and Cpt1a $(40,41)$. The observed increase in hepatic ABCA1 is consistent with a role for anti-miR33 in augmenting HDL biogenesis in the liver, and this is supported by our finding of an increase in apoA1 recovered from the HDL fraction. Furthermore, characterization of the HDL from anti-miR33-treated mice showed that a greater proportion of apoA1 and apoE was associated with larger HDL particles, consistent with the notion that the HDL in these mice is being lipidated from cells in the periphery - a function of RCT. Notably, the cholesterol ester content of the antimiR-33 HDL was also increased, and recent studies have shown that enrichment of HDL with apoE may enhance its ability to promote cholesterol efflux, which was attributed in part to the ability of apoE to activate lecithin:cholesterol acyltransferase, which converts free cholesterol to cholesteryl ester, thereby creating a gradient for free cholesterol efflux from cells to HDL (42-44).

The static measurement of HDL has inherent limitations, as several recent studies indicate that HDL may become dysfunctional or even proinflammatory (45). RCT - the efflux of excess cellular cholesterol from peripheral tissues and its return to the liver for excretion - is believed to be a critical mechanism by which HDL exerts its protective effects on atherosclerosis. Thus, to test the functionality of HDL generated in the setting of anti-miR33 treatment, we performed an in vivo RCT assay that measures the integrated rate of movement of ${ }^{3} \mathrm{H}$-cholesterol from macrophages to the serum, liver, and feces. We found that anti-miR33 treatment increases the flux of cholesterol from cholesterol-loaded macrophages to all 3 of these compartments and results in an $82 \%$ increase in fecal ${ }^{3} \mathrm{H}$-sterol excretion. Notably, this increase in RCT may reflect dual actions of anti-miR33: its ability to increase HDL biogenesis in the liver and its ability to directly promote the efflux of cholesterol from arterial macrophage foam cells by increasing expression of ABCA1 in these cells. While it is not known whether the anti-miR-33 is directly delivered to the arterial wall or taken up by circulating monocytes and delivered to plaques, the ability to target lesional macrophages makes anti-miR33 2' $\mathrm{F} / \mathrm{MOE}$ oligonucleotide an attractive potential therapeutic agent, as the collective outcome of numerous studies has shown that the rate of macrophage RCT has a greater influence on the degree of atherosclerosis than the levels of HDL-C $(45,46)$.

Although studies in animal models have shown that raising circulating HDL by overexpression of apoA1 or infusion of recombinant HDL retards plaque development $(3-5,8)$, the impact of $\mathrm{HDL}$ on regression of atherosclerosis is of great interest because of the important clinical implications. Thus, we chose to test the effects of anti-miR33 on atherosclerosis in a model of advanced disease. We first established atherosclerosis in $\mathrm{Ldll}^{-/}$- mice by feed- 
A 2'F/MOE anti-miR

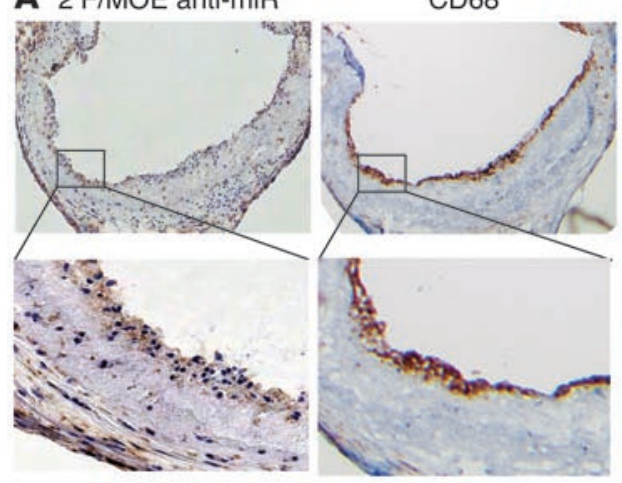

B

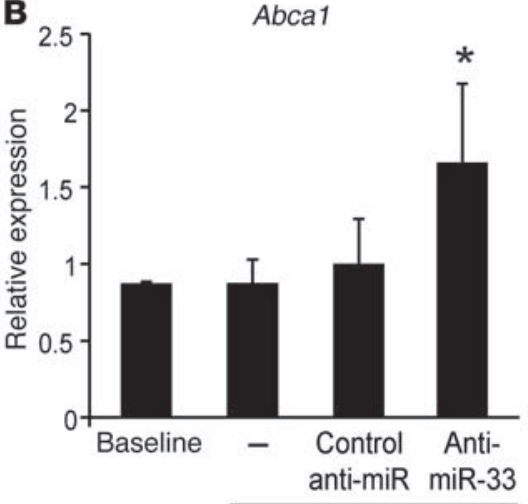

Treatment
C - Mmu-miR-33 seed heptamer-matched genes Non-seed-matched genes

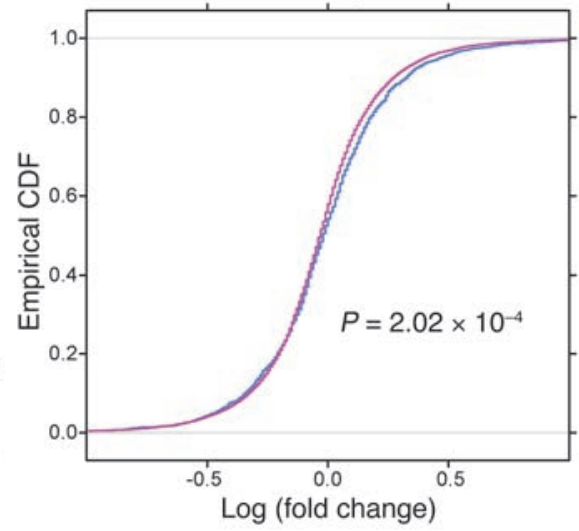

Figure 6

Anti-miR oligonucleotides reach plaque macrophages and alter target gene expression. (A) Immunohistochemical detection of the phosphorothioate backbone of the 2'F/MOE oligonucleotides (left panel) and CD68 (right panel) in serial sections of the aortic sinus. Original magnification: ×50 (top images); ×200 (lower images). (B) Expression of Abca1 mRNA in plaque CD68+ macrophages isolated by laser-capture microdissection and analyzed by qRT-PCR. PBS treatment is indicated by the dash. ${ }^{*} P \leq 0.05$, compared with all other groups. (C) Gene expression profiling was performed on mRNA of lesional macrophages isolated by laser-capture microdissection, and a CDF analysis was performed. CDF analysis showed a significant enrichment in the expression of genes containing miR-33 binding sites in anti-miR33-treated mice (blue line) compared with that in control anti-miR-treated mice (pink line).

ing them a WD for 14 weeks. Mice were then switched to a chow diet, simulating aggressive lipid lowering and reducing total cholesterol levels, which halts the progression of atherosclerosis but alone does not induce its regression. In this setting, treatment with anti-miR33 for 4 weeks caused a $35 \%$ reduction in lesion size and plaque macrophage and lipid content. In addition, plaques from anti-miR33-treated mice showed a 2-fold increase in collagen content, indicating an increase in lesion remodeling and plaque stability. These results suggest that anti-miR33 treatment in combination with lipid-lowering therapy would have an additive beneficial outcome on both lipid profile and plaque environment.

Numerous laboratories have shown that cholesterol loading of macrophages is proinflammatory, and recent in vitro and in vivo studies have established that cholesterol efflux to HDL or apoA1 dampens this inflammation $(8,38,47)$. Gene expression profiling of macrophages isolated by laser microdissection of plaques from anti-miR33 -treated and control anti-miR-treated mice revealed that in addition to reducing macrophage lipid content, antimiR33 treatment altered the inflammatory state of these cells. Lesional macrophages from anti-miR33-treated mice showed a switch from a proinflammatory M1 state characterized by iNOS and TNF- $\alpha$ expression to the more reparative M2 phenotype characterized by Arg1 and IL-10 expression. Notably, both Arg1 and IL-10 contain a single miR-33 binding site in their 3' UTRs, and, as such, the increase in Arg1 and Il10 mRNA in lesional macrophages of anti-miR-33-treated mice may represent direct derepression of these target genes by anti-miR-33. However, the increases in Arg1 and IL-10 expression are also consistent with recent studies from our group in other models of atherosclerosis regression, which showed that raising HDL-C or dramatically lowering LDL-C led to a depletion in M1 markers and an enrichment in markers of M2 macrophages $(8,38,48)$, which may indicate that these changes are hallmarks of regressing lesions. A major consequence of the switch from the M1 to M2 state is a diversion of $\mathrm{L}$-arginine metabolism from the iNOS pathway to the arginase 1 pathway that promotes collagen synthesis $(49,50)$. Thus, whether through direct derepression of miR-33 targets or HDL-mediated dampening of inflammation, this switch of macrophage phenotype to the M2 state by anti-miR33 treatment may both reduce lesional inflammation and contribute to plaque stabilization by increasing collagen synthesis.

Consistent with the atheroprotective effects of anti-miR33 shown herein, studies aimed at elucidating the impact of ABCA1 on atherosclerosis have been generally supportive of a protective role for ABCA1. Macrophage-specific deletion of ABCA1 resulted in an increase in atherosclerosis, whereas overexpression of $A B C A 1$ in macrophages provided protection from disease $(51,52)$. However, the effects of whole body transgenic overexpression of ABCA1 have been less clear. Despite an increase in circulating HDL similar in magnitude to the one observed in our study, transgenic overexpression of ABCA1 resulted in an accumulation of non-HDL lipoproteins and an increase in atherogenesis (53-55). Although the reasons for these results are not entirely clear, the authors speculated that this may be linked to reduced catabolism of HDL. By contrast, we find that antimiR33 treatment, which raises ABCA1 in both the liver and plaque macrophages, promotes the regression of atherosclerosis.

In the last decades, much progress has been made in understanding the molecular mechanisms that regulate cholesterol metabolism, and further understanding of how miRNAs are integrated into this complex genetic network may reveal novel therapeutic targets in this area. Previous studies have shown that antagonism of miR-122, the most abundantly expressed miRNA in the liver, results in sustained reduction in total plasma cholesterol in both the LDL and HDL fractions $(56,57)$. In addition, miR-122 antagonism in mice fed a highfat diet improved liver steatosis by reducing liver triglyceride content and increasing the rate of fatty acid $\beta$-oxidation (56). Despite these beneficial effects, the therapeutic potential of anti-miR122 is limited by a current lack of understanding of the direct gene targets by which anti-miR122 mediates these effects. It has been speculated that antimiR122 influences an unidentified transcriptional repressor of cho- 
A

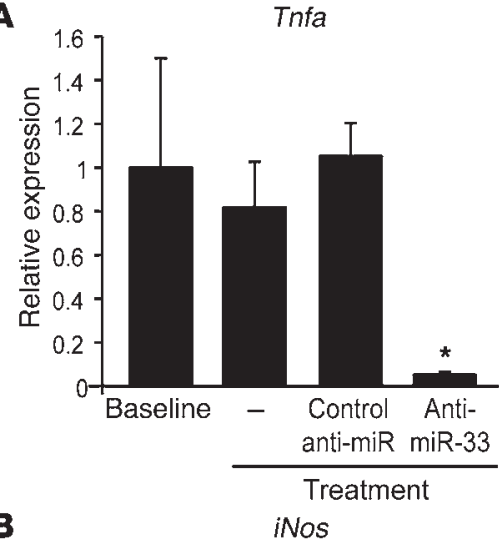

B

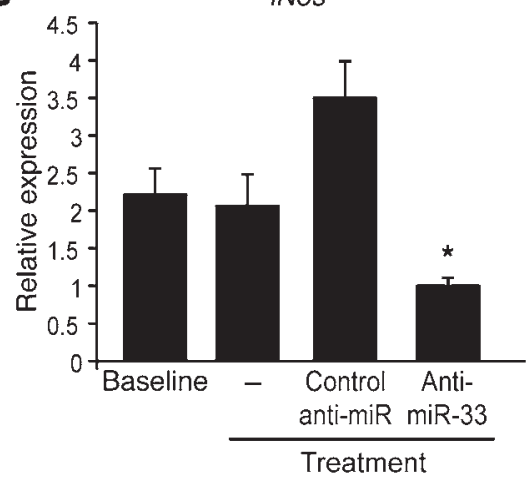

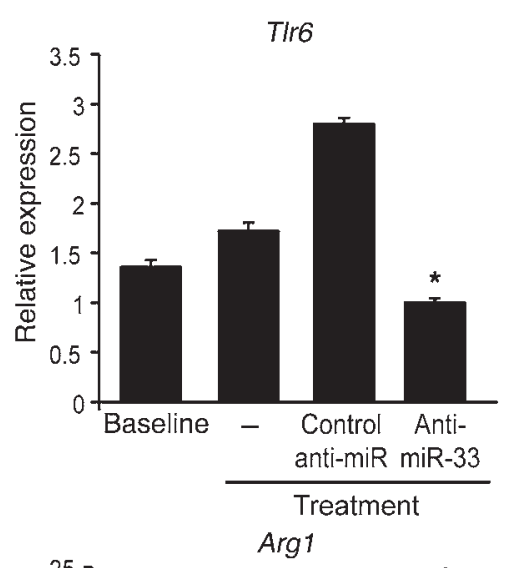

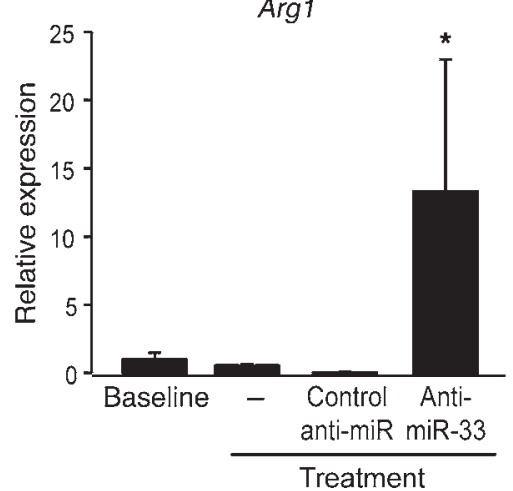

$\operatorname{Tr} 13$
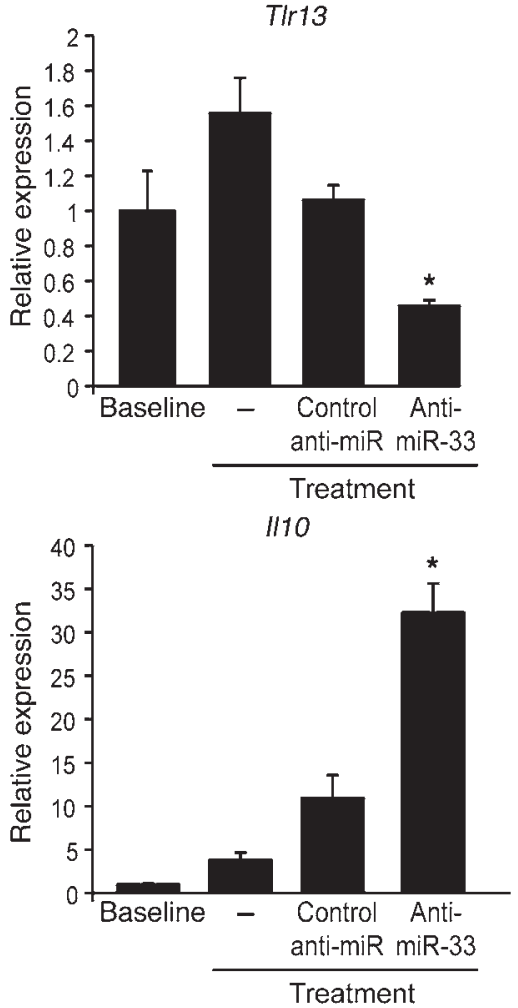

Figure 7

Macrophages from anti-miR33-treated plaques show reduced inflammatory gene expression. Lesional CD68+ macrophages were laser captured from aortic sinus lesions of $L d l r^{-1}$ mice receiving the indicated treatment, and mRNA was isolated. Expression of $(\mathbf{A})$ inflammatory genes $(T n f a$, TIr6, and TIr13) and (B) M1 and M2 markers (iNos, Arg1, and I/10) was analyzed by qRT-PCR. PBS treatment is indicated by the dash. Data are the mean expression levels from 4 mice per group \pm SEM. ${ }^{*} P<0.05$, compared with baseline.

lesterol biosynthesis or fatty acid metabolism pathways, and further study will be needed to clarify both the molecular mechanisms of its actions and the full extent of its effects. By contrast, our studies of miR33 show that it specifically targets genes involved in HDL biogenesis and cholesterol efflux ( $A B C A 1$ and $A B C G 1)$, and its antagonism results in direct upregulation of these pathways and enhanced cholesterol removal from peripheral tissues, leading to an overall improvement in RCT and atherosclerosis.

The statin class of HMG-CoA reductase inhibitors have revolutionized the treatment of atherosclerotic cardiovascular disease through their ability to aggressively lower LDL-C; however, the outcomes of large-scale clinical trials have shown that they only reduce subsequent cardiovascular events by $20 \%-40 \%(58,59)$. In this patient population, HDL remains an independent predictor of cardiovascular risk (60). Pharmacologic agents currently in clinical use that increase HDL-C, such as fibrates and niacin, have been associated with decreased cardiovascular events, suggesting that HDL raising may be an effective therapy to reduce residual disease burden $(61,62)$. Promising studies in humans demonstrating that HDL infusion regresses atherosclerosis have continued to fuel the search for suitable therapeutic targets to raise $\operatorname{HDL}(11,63)$. Inhibitors of cholesterol ester transfer protein, which potently raise HDL levels and lower LDL levels, are currently the most promising drug candidates $(17,64)$. However, there has been concern that this strategy might lead to the formation of "dysfunctional" HDL that is unable to promote cholesterol efflux from macrophage foam cells or that there might be a block in RCT from peripheral tissues to the liver (65-67). Our study in $\mathrm{Ldlr}^{-/}$mice, showing that anti-miR33 increases circulating HDL, enhances the RCT pathway, and regresses established atherosclerosis, indicates that antagonism of this microRNA might be a promising clinical approach for raising HDL in the treatment of cardiovascular disease.

\section{Methods}

Mice. All experiments were approved by the New York University School of Medicine Institutional Animal Care and Use Committee. $\mathrm{Ldlr}^{-/-}$mice were weaned at 4 weeks of age and placed on a high-fat diet $(21 \%$ [wt/wt] fat, $0.3 \%$ cholesterol; Research Diets) for 14 weeks, at which point mice were either sacrificed (baseline) or switched to chow diet for 4 weeks. Coincident with the switch to chow diet, mice were randomized into 3 groups $(n=15$ mice): no treatment (PBS), 2'F/MOE control anti-miR (TTATCGCCATGTCCAATGAGGCT) oligonucleotide, or 2'F/MOE anti-miR33 (TGCAATGCAACTACAATGCAC) oligonucleotide (Regulus Therapeutics). Mice received 2 subcutaneous injections of $10 \mathrm{mg} / \mathrm{kg}$ anti-miR (or an equivalent volume of PBS) the first week, spaced 2 days apart, and weekly injections of $10 \mathrm{mg} / \mathrm{kg}$ anti-miR (or PBS) thereafter for 4 weeks. After the second injection of anti-miR, mice were monitored for inflammation by measurement of serum IL- 6 and MCP- 1 by ELISA (eBioscience). At sacrifice, mice were anesthetized with isoflurane and exsanguinated by cardiac puncture. Mice were perfused with PBS, followed by $10 \%$ sucrose in PBS. Aortic roots were embedded in OCT medium and frozen immediately, and liver tissue was snap-frozen under liquid nitrogen and stored at $-80^{\circ} \mathrm{C}$. 
Plasma lipoprotein analysis. Plasma was collected at sacrifice, and total cholesterol was assayed (1:5 dilution) using the Cholesterol-E Kit (Wako) as described previously (28). For FPLC analysis, $300 \mu \mathrm{l}$ pooled plasma ( $n=8$ mice total) was separated on a Superose column (Amersham) at a flow rate of $0.4 \mathrm{ml} / \mathrm{min}$ as described previously (28). Fractions were collected and analyzed for total cholesterol content using the Cholesterol-E Kit. For HDL measurements, apoBcontaining lipoproteins were precipitated by the phosphotungstate-magnesium method, and HDL-C was measured using either the HDL Cholesterol Kit (Wako) or the Amplex Red Cholesterol Assay (Invitrogen) (28).

RNA isolation and quantitative PCR. Liver tissue was homogenized using the Bullet Blender Tissue Homogenizer (Next Advance), and total RNA was extracted using TRIzol Reagent (Invitrogen). RNA integrity was verified using the Agilent Biolanalzyer prior to use. For miRNA measurements, $1 \mu \mathrm{g}$ total RNA was reverse transcribed using the RT ${ }^{2}$ First Strand Synthesis Kit (SABiosciences), and miR-33 was detected using specific primers to mmumiR33 and normalized to U6 small RNA (SABiosciences) as described previously (28). For all other analysis, $1 \mu \mathrm{g}$ total RNA was reverse transcribed using iScript cDNA Synthesis Kit (Bio-Rad), and gene expression was measured by qRT-PCR and normalized to GAPDH as described previously $(28,68)$.

Western blotting. Protein was extracted from liver using the Bullet Blender in RIPA buffer, according to the manufacturer's protocol. For detection of ABCA 1 and ABCG1, $40 \mu$ g protein was separated on a $6 \%$ SDS-PAGE gel and transferred to nitrocellulose. Membranes were incubated overnight with antibodies to ABCA1 or ABCG1 (Abcam), and proteins were visualized using appropriate secondary antibodies conjugated to IR-dyes (Rockland) and scanned using the Odyssey Imaging System (Licor) as described previously (28). ABCA1 and ABCG1 protein expression was quantified and normalized to tubulin detected using CAT\#T6074 (Tubulin antibody) from Sigma-Aldrich. For detection of apoA1 and apoE in HDL-containing FPLC fractions, $10 \mu \mathrm{l}$ of pooled fractions were separated on a 10\% SDS-PAGE gel and transferred to PVDF. Membranes were incubated overnight with antibodies to apolipipoprotein A1 (apoA1) (Abcam) or apoE (R\&D Systems), and antibody reactivity was detected as described above.

In vivo RCT assay. Bone marrow-derived macrophages were prepared from C57BL/ 6 mice as previously described (68). Bone marrow was isolated, and cells were plated overnight in DMEM supplemented with 10\% FBS and 15\% L-929 conditioned media. Non-adherent cells were removed and cultured for an additional 6 days to allow for macrophage differentiation. For RCT assays, bone marrow-derived macrophage (BMDMs) were washed twice and incubated with $37.5 \mu \mathrm{g} / \mathrm{ml}$ acetylated LDL (acLDL) and $5 \mu \mathrm{Ci} / \mathrm{ml}^{3} \mathrm{H}$-cholesterol for 24 hours as described previously $(35,36)$. Cells were resuspended in ice-cold DMEM, and 2-3 $\times 10^{6}$ cells were injected subcutaneously into individually housed mice fed a WD diet and treated with either control antimiR or anti-miR33 for 4 weeks as described above. Prior to injection, an aliquot of cells was counted using liquid scintillation counting to measure baseline radioactivity. Blood was obtained by saphenous vein puncture at 6 and 24 hours after BMDM injection and by cardiac puncture after 48 hours at sacrifice. An aliquot of plasma was used for liquid scintillation counting immediately at each time point. Feces were collected for 24 and 48 hours after injection and homogenized in $50 \% \mathrm{NaOH}$ overnight, after which an aliquot was used for liquid scintillation counting. At sacrifice, liver samples were collected and incubated with hexane/isopropanol (3:2) for 48 hours and then dried overnight. Lipids were resolubilized in liquid scintillation fluid, and radioactivity was counted. RCT to plasma, feces, and liver was calculated as a percentage of total radioactivity injected at baseline.

Atherosclerosis analysis. Hearts embedded in OCT were sectioned through the aortic root $(8 \mu \mathrm{m})$ and stained with hematoxylin and eosin for lesion quantification or used for immunohistochemical analysis as previously described $(69,70)$. For morphometric analysis of lesions, 16 sections per mouse were imaged, spanning the entire aortic root, and lesions were quantified using
iVision Software. For collagen analysis, 10 sections per mouse were stained with Picrosirius Red and imaged under polarized light using a Zeiss Axioplan microscope. For detection of neutral lipid, oil red O staining was performed as previously described $(69,70)$. For macrophage analysis, 10 sections per mouse were incubated with an anti-CD68 antibody (rat anti-mouse CD68, 1:500; Serotec) and a secondary antibody conjugated to biotin (1:500), and antibody reactivity was visualized using the Vectastain ABC Elite Kit (Vector Laboratories) and diaminobenzidine (DAB; Sigma-Aldrich). For detection of 2'F/MOE oligonucleotides, frozen sections were fixed in neutral buffered formalin at room temperature and treated with Dako Dual Endogenous Enzyme-Blocking Reagent (DAKO) for 5 minutes. Slides were rinsed in PBS and blocked with $5 \%$ normal donkey serum, followed by incubation with primary antibodies raised against the phosphorothioate backbone of the 2'F/MOE oligonucleotides or a control antibody for 1 hour. Slides were incubated with HRP-conjugated donkey anti-rabbit secondary antibody for 30 minutes, and immunoreactivity was visualization with DAB substrate (DAKO).

Laser-capture microdissection. Laser-capture microdissection was performed using a PixCell II instrument (Arcturus Bioscience) as previously described $(71,72)$. To visualize $\mathrm{CD} 68^{+}$cells, a guide slide was prepared by staining for CD68 as described above. Cells corresponding to $\mathrm{CD}^{+} 8^{+}$area in serial sections were collected, and RNA was extracted using the Arcturus Picopure RNA Isolation Kit. Total RNA was amplified using the Ovation WT Pico Amp Kit (NuGen), purified using Qiaquick PCR Purification Kit (Qiagen), and used for quantitative PCR as described above.

Affymetrix gene array analysis. mRNA from macrophages collected by lasercapture microdissection was profiled for expression on Affymetrix Mouse 4302.0 arrays in quadruplicates. Macrophages were derived from 3 groups of mice: (a) anti-miR33-treated mice, (b) control anti-miR-treated mice, and (c) untreated mice. Microarray data were Robust Multichip Average (RMA) normalized (73), $\log _{2}$ transformed, and quality controlled by principal component analysis. The gene-level array data were then compared between treated versus untreated groups by 1-way ANOVA. The genes were then split between those containing one or more mir-33 seed-matched heptamers (nucleotides 1-7 or 2-8) in their $3^{\prime}$ UTRs and those that contained none. The R package (http://www.r-project.org/) was used to compute the CDF for the fold changes of these genes. Statistical significance of the shift between these 2 populations was determined by using a 1-sided Kolmogorov-Smirnov test. The microarray data discussed have been deposited in NCBI's Gene Expression Omnibus and are accessible through GEO accession number GSE28783 (http://www.ncbi.nlm.nih.gov/geo/query/acc.cgi?acc=GSE28783).

Statistics. For atherosclerosis and immunohistochemical analyses, all comparisons were made using a 1-way ANOVA $(P \leq 0.05)$, and data are expressed as mean \pm SEM, unless otherwise noted.

\section{Acknowledgments}

This work was supported by grants from the NIH (R01AG020255 and R01HL108182 to K.J. Moore; R01HL084312 and P01HL098055 to E.A. Fisher; R00 HL088528 to R.E. Temel; R01HL107953 to C. Fernandez-Hernando), the Canadian Institutes of Health Research (to K.J. Rayner), and the American Heart Association (SDG-0835481N to Y. Suarez; 0835585D to C. Fernandez-Hernando).

Received for publication February 1, 2011, and accepted in revised form April 27, 2011.

Address correspondence to: Kathryn J. Moore, New York University School of Medicine, 522 First Avenue, Smilow 705, New York, New York 10016, USA. Phone: 212.263.9259; Fax: 212.263.9115; E-mail: Kathryn.moore@nyumc.org. 
1. Castelli WP, et al. HDL cholesterol and other lipids in coronary heart disease. The cooperative lipoprotein phenotyping study. Circulation. 1977; 55(5):767-772

2. Wilson PW. High-density lipoprotein, low-density lipoprotein and coronary artery disease. Am J Cardiol. 1990;66(6):7A-10A.

3. Rubin EM, Krauss RM, Spangler EA, Verstuyft JG, Clift SM. Inhibition of early atherogenesis in transgenic mice by human apolipoprotein AI. Nature. 1991; 353(6341):265-267.

4. Paszty C, Maeda N, Verstuyft J, Rubin EM. Apolipoprotein AI transgene corrects apolipoprotein E deficiency-induced atherosclerosis in mice. J Clin Invest. 1994;94(2):899-903.

5. Plump AS, Scott CJ, Breslow JL. Human apolipoprotein A-I gene expression increases high density lipoprotein and suppresses atherosclerosis in the apolipoprotein E-deficient mouse. Proc Natl Acad SciU S A. 1994;91(20):9607-9611.

6. Choudhury RP, et al. High-density lipoproteins retard the progression of atherosclerosis and favorably remodel lesions without suppressing indices of inflammation or oxidation. Arterioscler Thromb Vasc Biol. 2004;24(10):1904-1909.

7. Tangirala RK, Tsukamoto K, Chun SH, Usher D, Pure E, Rader DJ. Regression of atherosclerosis induced by liver-directed gene transfer of apolipoprotein A-I in mice. Circulation. 1999; 100(17):1816-1822.

8. RongJX, et al. Elevating high-density lipoprotein cholesterol in apolipoprotein E-deficient mice remodels advanced atherosclerotic lesions by decreasing macrophage and increasing smooth muscle cell content. Circulation. 2001;104(20):2447-2452.

9. Shah PK, et al. High-dose recombinant apolipoprotein A-I(milano) mobilizes tissue cholesterol and rapidly reduces plaque lipid and macrophage content in apolipoprotein e-deficient mice. Potential implications for acute plaque stabilization. Circulation. 2001;103(25):3047-3050.

10. Badimon JJ, Badimon L, Fuster V. Regression of atherosclerotic lesions by high density lipoprotein plasma fraction in the cholesterol-fed rabbit. J Clin Invest. 1990;85(4):1234-1241.

11. Nissen SE, et al. Effect of recombinant ApoA-I Milano on coronary atherosclerosis in patients with acute coronary syndromes: a randomized controlled trial. Jama. 2003;290(17):2292-2300.

12. Brooks-Wilson A, et al. Mutations in ABC1 in Tangier disease and familial high-density lipoprotein deficiency. Nat Genet. 1999;22(4):336-345.

13. Bodzioch M, et al. The gene encoding ATP-binding cassette transporter 1 is mutated in Tangier disease. Nat Genet. 1999;22(4):347-351.

14. Oram JF, Vaughan AM. ATP-Binding cassette cholesterol transporters and cardiovascular disease. Circ Res. 2006;99(10):1031-1043.

15. Kennedy MA, et al. ABCG1 has a critical role in mediating cholesterol efflux to HDL and preventing cellular lipid accumulation. Cell Metab. 2005; 1(2):121-131.

16. Wang N, Lan D, Chen W, Matsuura F, Tall AR. ATP-binding cassette transporters G1 and G4 mediate cellular cholesterol efflux to high-density lipoproteins. Proc Natl Acad Sci U S A. 2004; 101(26):9774-9779.

17. Tall AR, Yvan-Charvet L, Terasaka N, Pagler T, Wang N. HDL, ABC transporters, and cholesterol efflux: implications for the treatment of atherosclerosis. Cell Metab. 2008;7(5):365-375.

18. Wang X, et al. Macrophage ABCA1 and ABCG1, but not SR-BI, promote macrophage reverse cholesterol transport in vivo. J Clin Invest. 2007; 117(8):2216-2224.

19. Out R, et al. Combined deletion of macrophage $\mathrm{ABCA} 1$ and $\mathrm{ABCG} 1$ leads to massive lipid accumulation in tissue macrophages and distinct athero- sclerosis at relatively low plasma cholesterol levels. Arterioscler Thromb Vasc Biol. 2008;28(2):258-264.

20. Yvan-Charvet L, et al. Combined deficiency of ABCA1 and ABCG1 promotes foam cell accumulation and accelerates atherosclerosis in mice. J Clin Invest. 2007;117(12):3900-3908.

21 . Adorni MP, et al. The roles of different pathways in the release of cholesterol from macrophages.J Lipid Res. 2007;48(11):2453-2462.

22. Tontonoz P, Mangelsdorf DJ. Liver X receptor signaling pathways in cardiovascular disease. $\mathrm{Mol}$ Endocrinol. 2003;17(6):985-993.

23. Joseph SB, et al. Synthetic LXR ligand inhibits the development of atherosclerosis in mice. Proc Natl Acad Sci U S A. 2002;99(11):7604-7609.

24. Verschuren L, de Vries-van der Weij J, Zadelaar S, Kleemann R, Kooistra T. LXR agonist suppresses atherosclerotic lesion growth and promotes lesion regression in apoE*3Leiden mice: time course and mechanisms. J Lipid Res. 2009;50(2):301-311.

25. Feig JE, et al. LXR promotes the maximal egress of monocyte-derived cells from mouse aortic plaques during atherosclerosis regression. J Clin Invest. 2010; 120(12):4415-4424.

26. Naik SU, et al. Pharmacological activation of liver $\mathrm{X}$ receptors promotes reverse cholesterol transport in vivo. Circulation. 2006;113(1):90-97.

27. Bradley MN, et al. Ligand activation of LXR beta reverses atherosclerosis and cellular cholesterol overload in mice lacking LXR alpha and apoE. J Clin Invest. 2007;117(8):2337-2346.

28. Rayner KJ, et al. MiR-33 contributes to the regulation of cholesterol homeostasis. Science. 2010; 328(5985):1570-1573.

29. Najafi-Shoushtari SH, et al. MicroRNA-33 and the SREBP host genes cooperate to control cholesterol homeostasis. Science. 2010;328(5985):1566-1569.

30. Marquart TJ, Allen RM, Ory DS, Baldan A. miR33 links SREBP-2 induction to repression of sterol transporters. Proc Natl Acad Sci U S A. 2010; 107(27):12228-12232.

31. Davis S, et al. Potent inhibition of microRNA in vivo without degradation. Nucleic Acids Res. 2009; 37(1):70-77.

32. Elmen J, et al. LNA-mediated microRNA silencing in non-human primates. Nature. 2008; 452(7189):896-899.

33. Lanford RE, et al. Therapeutic silencing of microRNA-122 in primates with chronic hepatitis $C$ virus infection. Science. 2010;327(5962):198-201.

34. Henry S, Stecker K, Brooks D, Monteith D, Conklin B, Bennett CF. Chemically modified oligonucleotides exhibit decreased immune stimulation in mice. J Pharmacol Exp Ther. 2000;292(2):468-479.

35. Zhang Y, Da Silva JR, Reilly M, Billheimer JT, Rothblat GH, Rader DJ. Hepatic expression of scavenger receptor class B type I (SR-BI) is a positive regulator of macrophage reverse cholesterol transport in vivo. J Clin Invest. 2005;115(10):2870-2874.

36. Wang X, et al. Macrophage ABCA1 and ABCG1, but not SR-BI, promote macrophage reverse cholesterol transport in vivo. J Clin Invest. 2007; 117(8):2216-2224.

37. Geary RS. Antisense oligonucleotide pharmacokinetics and metabolism. Expert Opin Drug Metab Toxicol. 2009;5(4):381-391.

38. Feig JE, et al. HDL promotes rapid atherosclerosis regression in mice and alters inflammatory properties of plaque monocyte-derived cells. Proc Natl Acad Sci U S A. 2011;108(17):7166-7171.

39. Horie T, et al. MicroRNA-33 encoded by an intron of sterol regulatory element-binding protein 2 (Srebp2) regulates HDL in vivo. Proc Natl Acad Sci US A. 2010;107(40):17321-17326.

40. Gerin I, et al. Expression of miR-33 from an SREBP2 intron inhibits cholesterol export and fatty acid oxidation. J Biol Chem. 2010;285(44):33652-33661.

41. Dávalos A, et al. miR-33a/b contribute to the regulation of fatty acid metabolism and insulin signaling [published online ahead of print May 16, 2011]. Proc Natl Acad Sci U S A. doi:10.1073/ pnas. 1102281108 .

42. Yvan-Charvet L, et al. Cholesterol efflux potential and antiinflammatory properties of highdensity lipoprotein after treatment with niacin or anacetrapib. Arterioscler Thromb Vasc Biol. 2010; 30(7):1430-1438.

43. Matsuura F, Wang N, Chen W, Jiang XC, Tall AR. HDL from CETP-deficient subjects shows enhanced ability to promote cholesterol efflux from macrophages in an apoE- and ABCG1-dependent pathway. J Clin Invest. 2006;116(5):1435-1442.

44. Innerarity TL, Pitas RE, Mahley RW. Modulating effects of canine high density lipoproteins on cholesteryl ester synthesis induced by beta-very low density lipoproteins in macrophages. Possible in vitro correlates with atherosclerosis. Arteriosclerosis. 1982;2(2):114-124.

45. Khera AV, et al. Cholesterol efflux capacity, highdensity lipoprotein function, and atherosclerosis. N Engl J Med. 2011;364(2):127-135.

46. Rader DJ, Alexander ET, Weibel GL, Billheimer J, Rothblat GH. The role of reverse cholesterol transport in animals and humans and relationship to atherosclerosis. J Lipid Res. 2009;50 suppl:S189-S194.

47. Sun Y, et al. Free cholesterol accumulation in macrophage membranes activates Toll-like receptors and p38 mitogen-activated protein kinase and induces cathepsin K. Circ Res. 2009;104(4):455-465.

48. Feig JE, et al. Reversal of hyperlipidemia with a genetic switch favorably affects the content and inflammatory state of macrophages in atherosclerotic plaques. Circulation. 2011;123(9):989-998.

49. Gordon S. Alternative activation of macrophages. Nat Rev Immunol. 2003;3(1):23-35.

50. Mills CD. Macrophage arginine metabolism to ornithine/urea or nitric oxide/citrulline: a life or death issue. Crit Rev Immunol. 2001;21(5):399-425.

51. Aiello RJ, et al. Increased atherosclerosis in hyperlipidemic mice with inactivation of ABCA1 in macrophages. Arterioscler Thromb Vasc Biol. 2002; 22(4):630-637.

52. Van Eck M, et al. Macrophage ATP-binding cassette transporter A1 overexpression inhibits atherosclerotic lesion progression in low-density lipoprotein receptor knockout mice. Arterioscler Thromb Vasc Biol. 2006;26(4):929-934.

53. Vaisman BL, et al. ABCA1 overexpression leads to hyperalphalipoproteinemia and increased biliary cholesterol excretion in transgenic mice. J Clin Invest. 2001;108(2):303-309.

54. Joyce CW, et al. ABCA1 overexpression in the liver of LDLr-KO mice leads to accumulation of proatherogenic lipoproteins and enhanced atherosclerosis. J Biol Chem. 2006;281(44):33053-33065.

55. Singaraja RR, et al. Increased ABCA1 activity protects against atherosclerosis. J Clin Invest. 2002;110(1):35-42.

56. Esau C, et al. miR-122 regulation of lipid metabolism revealed by in vivo antisense targeting. Cell Metab. 2006;3(2):87-98.

57. Elmen J, et al. Antagonism of microRNA-122 in mice by systemically administered LNA-antimiR leads to up-regulation of a large set of predicted target mRNAs in the liver. Nucleic Acids Res. 2008; 36(4):1153-1162.

58. Baigent C, et al. Efficacy and safety of cholesterollowering treatment: prospective meta-analysis of data from 90,056 participants in 14 randomised trials of statins. Lancet. 2005;366(9493):1267-1278.

59. Kearney PM, et al. Efficacy of cholesterol-lowering therapy in 18,686 people with diabetes in 14 randomised trials of statins: a meta-analysis. Lancet. 2008;371(9607):117-125.

60. Barter P, et al. HDL cholesterol, very low levels of LDL cholesterol, and cardiovascular events. NEngl 
JMed. 2007;357(13):1301-1310.

61. Taylor AJ, Kent SM, Flaherty PJ, Coyle LC, Markwood TT, Vernalis MN. ARBITER: Arterial Biology for the Investigation of the Treatment Effects of Reducing Cholesterol: a randomized trial comparing the effects of atorvastatin and pravastatin on carotid intima medial thickness. Circulation. 2002; 106(16):2055-2060.

62. Taylor AJ, Sullenberger LE, Lee HJ, Lee JK, Grace KA. Arterial Biology for the Investigation of the Treatment Effects of Reducing Cholesterol (ARBITER) 2: a double-blind, placebo-controlled study of extended-release niacin on atherosclerosis progression in secondary prevention patients treated with statins. Circulation. 2004;110(23):3512-3517.

63. Tardif JC, et al. Effects of reconstituted high-density lipoprotein infusions on coronary atherosclerosis: a randomized controlled trial. JAMA 2007;297(15):1675-1682.
64. Cannon CP, et al. Safety of anacetrapib in patients with or at high risk for coronary heart disease. N Engl J Med. 2010;363(25):2406-2415.

65. Rader DJ. Illuminating HDL--is it still a viable therapeutic target? $N$ Engl J Med. 2007; 357(21):2180-2183.

66. Tall AR. CETP inhibitors to increase HDL cholesterol levels. N Engl J Med. 2007;356(13):1364-1366.

67. Tall AR, Yvan-Charvet L, Wang N. The failure of torcetrapib: was it the molecule or the mechanism? Arterioscler Thromb Vasc Biol. 2007;27(2):257-260.

68. Stewart CR, et al. CD36 ligands promote sterile inflammation through assembly of a Toll-like receptor 4 and 6 heterodimer. Nat Immunol. 2010; 11(2):155-161.

69. Moore KJ, et al. Loss of receptor-mediated lipid uptake via scavenger receptor A or CD36 pathways does not ameliorate atherosclerosis in hyperlipidemic mice. J Clin Invest. 2005;115(8):2192-2201.
70. Manning-Tobin JJ, et al. Loss of SR-A and CD36 activity reduces atherosclerotic lesion complexity without abrogating foam cell formation in hyperlipidemic mice. Arterioscler Thromb Vasc Biol. 2009; 29(1):19-26.

71. Trogan E, Choudhury RP, Dansky HM, Rong JX, Breslow JL, Fisher EA. Laser capture microdissection analysis of gene expression in macrophages from atherosclerotic lesions of apolipoprotein E-deficient mice. Proc Natl Acad Sci U S A. 2002; 99(4):2234-2239.

72. Trogan E, Fisher EA. Laser capture microdissection for analysis of macrophage gene expression from atherosclerotic lesions. Methods Mol Biol. 2005; 293:221-231.

73. Bolstad BM, Irizarry RA, Astrand M, Speed TP. A comparison of normalization methods for high density oligonucleotide array data based on variance and bias. Bioinformatics. 2003;19(2):185-193. 